\title{
INVERSE AND MOORE-PENROSE INVERSE OF TOEPLITZ MATRICES WITH CLASSICAL HORADAM NUMBERS
}

\author{
Shougiang Shen, Weijun LiU ANd Lihua Feng
}

Abstract. For integers $s, k$ with $s \leqslant 0$ and $k \geqslant 0$, we define a class of lower triangular Toeplitz matrices $\mathscr{U}_{n}^{(s, k)}$ of type $(s, k)$, whose non-zero entries are the classical Horadam numbers $U_{i}^{(a, b)}$. In this paper, we derive a convolution formula containing the Horadam numbers. Using this formula, we obtain several combinatorial identities involving the Horadam numbers and the generalized Fibonacci numbers. In addition, we derive the inverse of the lower triangular Toeplitz matrix $\mathscr{U}_{n}^{(0, k)}$ and the Moore-Penrose inverse of the strictly lower triangular Toeplitz matrix $\mathscr{U}_{n}^{(s, k)}(s<0)$ by utilizing only the Horadam numbers.

Mathematics subject classification (2010): 15A09, 11B39, 05A19.

Keywords and phrases: Inverse, Moore-Penrose inverse, Horadam number, generalized Fibonacci number, Toeplitz matrix.

\section{REFERENCES}

[1] M. AKBULAK, D. BozKURT, On the norms of Toeplitz matrices involving Fibonacci and Lucas numbers, Hacet. J. Math. Stat. 37 (2008) 89-95.

[2] R. Chan, M. NG, Conjugate gradient methods for Toeplitz systems, SIAM Rev. 38 (1996) 427-482.

[3] P. CoUrRIEU, Fast computation of Moore-Penrose inverse matrices, Neural Information ProcessingLetters and Reviews 8 (2005) 25-29.

[4] S. FAlcón, Á. PlazA, The k-Fibonacci sequence and the Pascal 2-triangle, Chaos, Solitons Fract. 33 (2007) 38-49.

[5] S. Falcón, Á. Plaza, On k-Fibonacci numbers of arithmetic indexes, Appl. Math. Comput. 208 (2009) 180-185.

[6] A. B. Israel, T. N. E. Greville, Generalized Inverses: Theory and Applications, Second Ed., Springer, New York, 2003.

[7] T. Kailath, A. SAyed, Displacement structure: Theory and Applications, SIAM Rev. 37 (1995) 297-386.

[8] G.-Y. LEE, J.-S. KIM, S.-G. LEE, Factorizations and eigenvalues of Fibonacci and symmetric Fibonacci matrices, Fibonacci Quart. 40 (2002) 203-211.

[9] G.-Y. LEE, J.-S. KIM, S.-H. CHO, Some combinatorial identities via Fibonacci numbers, Discrete Appl. Math. 130 (2003) 527-534.

[10] M. Miladinović, P. Stanimirović, Singular case of generalized Fibonacci and Lucas matrices, J. Korean Math. Soc. 48 (2011) 33-48.

[11] S.-Q. SHEN, On the norms of Toeplitz matrices involving $k$-Fibonacci and $k$-Lucas numbers, Int. J. Contemp. Math. Science 7 (2012) 363-368.

[12] S.-Q. Shen, J.-J. HE, Moore-Penrose inverse of generalized Fibonacci matrix and its applications, Int. J. Comput. Math. 93 (2016) 1756-1770.

[13] P. Stanimirović, J. Nikolov, I. Stanimirović, A generalization of Fibonacci and Lucas matrices, Discrete Appl. Math. 156 (2008) 2606-2619.

[14] P. Stanimirović, M. Miladinović, Inversion of the generalized Fibonacci matrix by convolution, Int. J. Comput. Math. 88 (2011) 1519-1532. 
[15] K. Uslu, N. TAskara, H. Kose, The generalized k-Fibonacci and $k$-Lucas numbers, Ars Combinatoria 99 (2011) 25-32.

[16] Z. Zhang, Y. Zhang, The Lucas matrix and some combinatorial identities, Indian J. Pure Appl. Math. 38 (2007) 457-465. 